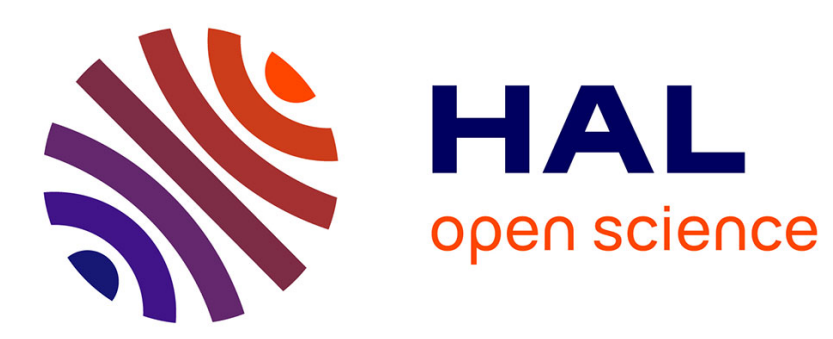

\title{
Vitesse des dislocations dans la glace dopée avec HF
}

\author{
C. Mai, J. Perez, J. Tatibouët, R. Vassoille
}

\section{To cite this version:}

C. Mai, J. Perez, J. Tatibouët, R. Vassoille. Vitesse des dislocations dans la glace dopée avec HF. Journal de Physique Lettres, 1978, 39 (17), pp.307-310. 10.1051/jphyslet:019780039017030700 . jpa00231504

\section{HAL Id: jpa-00231504 https://hal.science/jpa-00231504}

Submitted on 1 Jan 1978

HAL is a multi-disciplinary open access archive for the deposit and dissemination of scientific research documents, whether they are published or not. The documents may come from teaching and research institutions in France or abroad, or from public or private research centers.
L'archive ouverte pluridisciplinaire HAL, est destinée au dépôt et à la diffusion de documents scientifiques de niveau recherche, publiés ou non, émanant des établissements d'enseignement et de recherche français ou étrangers, des laboratoires publics ou privés. 


\title{
VITESSE DES DISLOCATIONS DANS LA GLACE DOPÉE AVEC HF
}

\author{
C. MAI, J. PEREZ, J. TATIBOUËT et R. VASSOILLE
}

Groupe d'Etudes de Métallurgie Physique et de Physique des Matériaux, INSA de Lyon $\left({ }^{*}\right)$, Bât. 502, 20, avenue A.-Einstein, 69621 Villeurbanne Cedex, France

(Reçu le 7 juin 1978, révisé le 6 juillet 1978, accepté le 12 juillet 1978)

\begin{abstract}
Résumé. - Les mesures de vitesse des dislocations par topographie $\mathrm{X}$ dans la glace dopée avec HF ont permis d'observer un comportement non-linéaire plus accentué que dans le cas de la glace pure et de mettre en évidence une accélération du mouvement des dislocations due aux effets de ce dopage. Les modèles théoriques basés sur une modification du désordre protonique grâce aux défauts rotationnels ne permettent pas d'expliquer l'ensemble des résultats expérimentaux. Nous sommes ainsi amenés à considérer le modèle reposant sur les hypothèses d'un cœur de dislocation non-cristallin et d'un mouvement coopératif des molécules d'eau. Les valeurs numériques calculées à partir de ce modèle sont compatibles avec les résultats expérimentaux.
\end{abstract}

\begin{abstract}
The velocity of individual dislocations in HF-doped ice single crystal has been measured by X-ray topography. The results show on the one hand an increase of the velocity and on the other hand, an increase of the non-linear dependence on shear stress compared to the velocity of dislocations in pure ice.

Theoretical models based on a process rate-limited by proton disorder cannot explain all the expe'rimental features. A new model, based on the cooperative movements of $\mathrm{H}_{2} \mathrm{O}$ molecules in noncrystalline dislocation cores, leads to numerical values which are in a good agreement with experimental results.
\end{abstract}

Introduction. - L'effét des impuretés telles que $\mathrm{NH}_{4} \mathrm{OH}, \mathrm{NH}_{4} \mathrm{~F}, \mathrm{HCl}, \mathrm{HF}, \ldots$ sur les propriétés mécaniques, en particulier le comportement plastique de la glace, est bien connu. On observe, dans le cas du dopage avec HF, par exemple, un effet d'adoucissement au cours des essais de traction (ou de compression) ou une augmentation de la vitesse de déformation pendant les essais de fluage. Par contre, d'autres impurétés telles que He peuvent introduire un faible effet de durcissement.

Des observations directes par topographie $\mathrm{X} d u$ comportement dynamique des dislocations dans la glace pure ont été réalisées $[1,2]$. Les récentes études sur la glace pure [3] montrent un comportement non-linéaire des dislocations vis-à-vis de la contrainte, d'autant plus net que la température est élevée.

Dans le cas de la glace dopée avec HF, Jones et Gilra [4] ont observé une forte augmentation de la densité des dislocations. Cependant, à notre connaissance, aucune observation directe du mouvement des dislocations dans la glace dopée lors de la déformation plastique n'a été réalisée.

$\left(^{*}\right)$ ERA № 463.
L'objet de ce travail est d'étudier l'influence du dopage avec HF sur le comportement dynamique des dislocations dans la glace $\mathrm{I}_{\mathrm{h}}$ monocristalline.

Etude expérimentale. - Les cristaux de glace sont obtenus soit par la méthode de Bridgman (Laboratoire de Glaciclogie de Grenoble, France) soit par la méthode de fusion de zone (Département de l'Environnement Ottawa, Canada).

Les monocristaux sont taillés mécaniquement puis polis chimiquement jusqu'aux dimensions $30 \times 6 \times 2 \mathrm{~mm}$. Ces échantillons sont ensuite recouverts d'une mince couche d'une solution à $5 \%$ de $\mathrm{HF}$ maintenue à une température de $263 \mathrm{~K}$ et ceci pendant 12 heures. Après polissage chimique de la surface, une partie de ces échantillons est analysée pour déterminer la concentration en HF par deux méthodes différentes :

- mesure du potentiel de Nernst par électrode spécifique après fusion de la glace dopée ;

- mesure du temps de relaxation par frottement intérieur [5].

La teneur en HF obtenue dans ces conditions est de l'ordre de $10 \mathrm{ppm}$. Après plusieurs jours dans 
l'enceinte froide où se font les mesures, la teneur globale en HF n'a pas varié.

Les mesures de vitesse des dislocations sont réalisées immédiatement après le dopage par la méthode topographie X en transmission. La longueur d'onde $K_{\alpha_{1}} \mathrm{du}$ molybdène est utilisée, ce qui donne un produit $\mu t<1 \quad(\mu$ : coefficient linéaire d'absorption de la glace, $t$ : épaisseur). La contrainte est appliquée par compression de l'échantillon directement sur le goniomètre de la chambre de Lang. Avant chaque série de mesures nous réalisons le topogramme de l'ensemble $\mathrm{du}$ cristal. Nous choisissons ensuite une section du cristal pour suivre le mouvement des dislocations, ce qui permet de réduire le temps d'exposition. Les mesures de vitesse sont faites sur des topogrammes obtenus par la technique de double exposition avant et après déformation.

L'ensemble des mesures couvre un domaine de température compris entre $251 \mathrm{~K}$ et $270 \mathrm{~K}$ et un domaine de contrainte de $0,2 \mathrm{à} 4 \times 10^{5} \mathrm{Nm}^{-2}$.

Résultats expérimentaux. - Rappelons les résultats obtenus sur la glace non dopée par la même méthode (Fig. 1) [3]. A basse température ( $T<255 \mathrm{~K}$ environ) la vitesse des dislocations est proportionnelle à la contrainte. Par contre, à plus haute température, cette loi linéaire n'est plus observée.

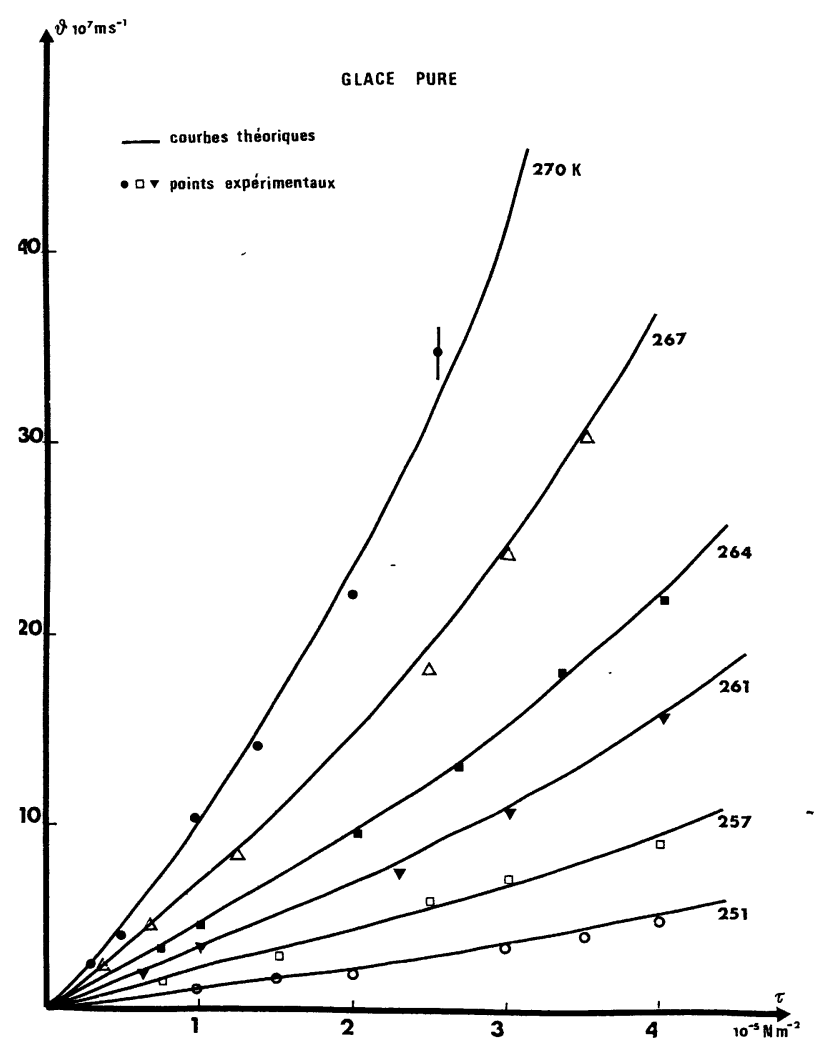

Fig. 1. - Vitesse des dislocations dans la glace pure en fonction de la contrainte réduite. Les courbes théoriques sont tracées à partir de la relation (2), [10].

[Dislocation velocity in pure ice versus resolved shear stress. The theoretical curves are calculated from the relation (2), [10].]

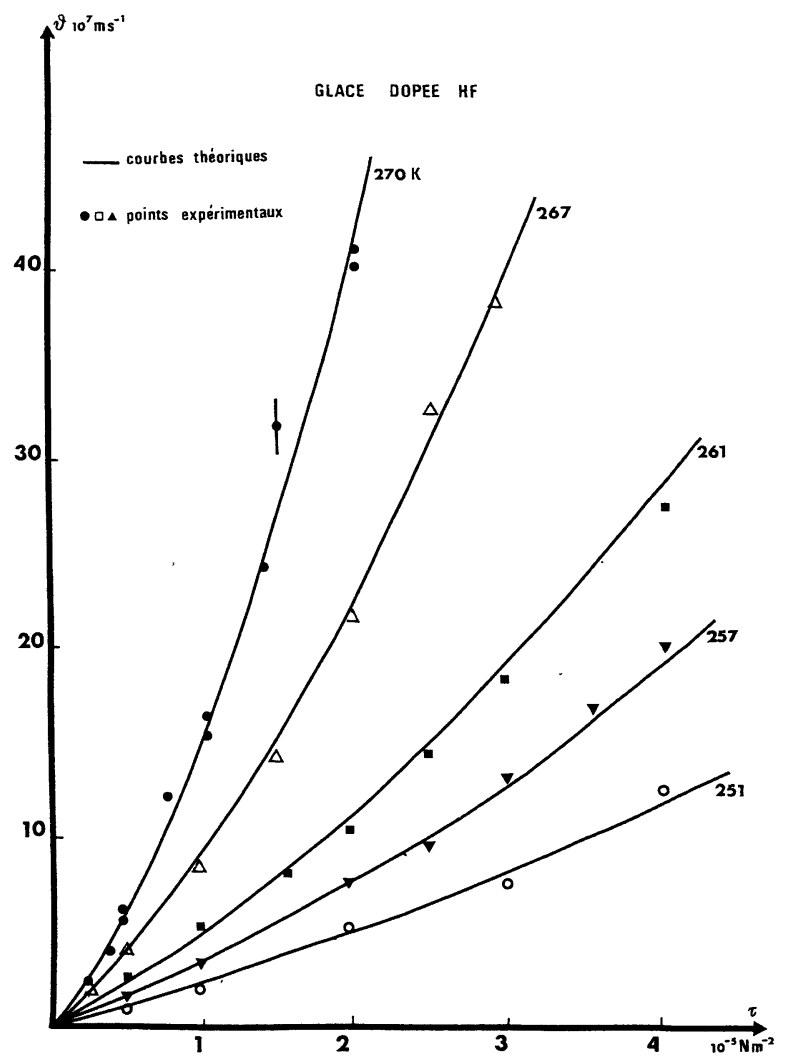

FIG. 2. - Vitesse des dislocations dans la glace dopée (10 ppm HF) en fonction de la contrainte réduite. Les courbes théoriques sont tracées à partir de la relation (2) en tenant compte de l'effet du dopage [14].

[Dislocation velocity in HF-doped ice versus resolved shear stress. The theoretical curves are calculated from the relation (2) taking into account the doping effect [14].]

Dans le cas de la glace dopée avec HF (Fig. 2) on observe d'une part un comportement non-linéaire des dislocations accentué dans tout le domaine de température et d'autre part, une vitesse plus élevée des dislocations. Avant de discuter plus en détail ces résultats expérimentaux, on peut dégager les observations suivantes :

a) dans le cas de la glace non dopée, les valeurs mesurées de la vitesse des dislocations sont très supérieures à celles issues des modèles théoriques basés sur la possibilité du réarrangement des protons grâce aux défauts rotationnels $[6,7,8]$;

b) le temps de relaxation $\tau_{\mathrm{R}}$ dans la formule (1) mesuré par frottement intérieur [5] est de l'ordre de 70 fois plus faible après le dopage de la glace avec HF $(\sim 10 \mathrm{ppm})$; or les vitesses mesurées dans la même glace dopée ne sont augmentées que d'un facteur de 1,5 à 2 ;

c) la non-linéarité (en fonction de la contrainte et de la température) est accentuée par dopage.

Discussion. - Nous allons maintenant discuter les points précédemment signalés portant sur les valeurs 
de la vitesse des dislocations et les effets de nonlinéarité vis-à-vis de la contrainte et de la température.

Rappelons tout d'abord, quelle peut être l'origine de la non-linéarité de la vitesse des dislocations. Si l'on considère les modèles théoriques classiques du comportement dynamique des dislocations dans la glace, basés sur une modification possible de l'arrangement des protons grâce aux défauts rotationnels $[6,7,8]$ on constate que, quel que soit le mécanisme supposé :

- diffusion de décrochements géométriques,

- mouvement d'arcs de dislocation décrits en termes de cordes flexibles,

on aboutit à une relation linéaire entre la vitesse des dislocations $v_{\mathrm{d}}$ et la contrainte $\tau$ :

$$
v_{\mathrm{d}}=\frac{A}{\tau_{\mathrm{r}}} \tau
$$

$A$ : constante

$\tau_{\mathrm{r}}$ : temps de relaxation liée au retournement des molécules d'eau.

Le calcul théorique à partir de cette relation donne une vitesse de l'ordre de $v_{\mathrm{d}} \simeq 50 \mathrm{~b} \mathrm{~s}^{-1}$. Or les mesures expérimentales des vitesses de dislocation, même à basse température c'est-à-dire dans le domaine où la vitesse est proportionnelle à la contrainte, donnent des valeurs :

$$
200 b^{-1} \leqslant v_{\mathrm{d}} \leqslant 900 \mathrm{bs}^{-1} .
$$

(Toutes les valeurs correspondent à $T=255 \mathrm{~K}$ et $\tau=10^{5} \mathrm{Nm}^{-2}$.)

La récente analyse de Whitworth [9] montre bien cette incompatibilité entre l'ensemble des résultats expérimentaux et les modèles théoriques du comportement dynamique des dislocations basés sur la possibilité du retournement des molécules d'eau grâce aux défauts rotationnels.

Un nouveau modèle reposant sur les hypothèses d'un cœur de dislocation non-cristallin et d'un mouvement coopératif des molécules d'eau a été propósé [10]. On obtient une relation du type :

$$
\begin{aligned}
v_{\mathrm{d}}= & \frac{4}{9 \pi\left(b r^{*}\right)^{3 / 2} \tau_{0}}\left(\frac{\tau}{G}\right)^{1 / 2} \times \\
& \times \exp \left[-\frac{2}{3}\left(\frac{E}{k T}\right)^{2}\right] \sinh \frac{\left(b r^{*}\right)^{3 / 2}(G \tau)^{1 / 2}}{2 k T}
\end{aligned}
$$

$\tau_{0}$ : inverse de la fréquence fondamentale des modes de vibration en translation (de l'ordre de $10^{-13} \mathrm{~s}$ [10])

$E$ : valeur la plus probable de l'énergie de liaison dans l'hypothèse d'une distribution linéaire des énergies de liaison [10]

$G$ : module de cisaillement (nous utiliserons dans les calculs la valeur $3,5 \times 10^{9} \mathrm{Nm}^{-2}$ )

$b$ : module du vecteur de Burgers des dislocations $\left(4,5 \times 10^{-10} \mathrm{~m}\right)$ $r^{*}$ : rayon du cœur étendu des dislocations ( $r^{*}$ augmente avec la température : $\sim 7 \AA$ à $250 \mathrm{~K}$ et $\sim 15 \AA$ à $270 \mathrm{~K}$ ).

Sans entrer dans le détail de ce modèle, on peut cependant en tirer les constatations suivantes :

- les valeurs théoriques de $v_{\mathrm{d}}$ sont comparables aux valeurs expérimentales,

- à basse température ou à faible contrainte la relation (2) se simplifie pour prendre une forme analogue à (1)

$$
v_{\mathrm{d}}=B \exp \left[-\frac{2}{3}\left(\frac{E}{k T}\right)^{2}\right] \tau
$$

$B$ : constante.

Par conséquent, la vitesse $v_{\mathrm{d}}$ varie dans ces conditions linéairement avec la contrainte. Cette linéarité disparaît lorsque la contrainte ou la température augmente. De plus, la non-linéarité est d'autant plus importante que la température est plus élevée.

Un tel modèle conduit donc à des données compatibles avec nos résultats expérimentaux [10, 14].

Nous devons donc maintenant confronter l'effet du dopage de la glace à ce dernier modèle. Si, comme nous l'avons vu, des essais mécaniques ont montré un effet d'adoucissement de la glace dopée avec HF [11], d'autres permettent de préciser que l'addition de HF entraîne à la fois une diminution de la hauteur du pic de traction (ou compression) et une décroissance du niveau de contrainte après le pic [12]. La théorie de Gilman et Johnston [13] permet d'attribuer le premier effet essentiellement à l'augmentation de la densité de dislocations due au dopage. Le deuxième effet, par contre, est principalement associé à l'augmentation de la vitesse des dislocations.

Cette augmentation de la vitesse des dislocations est d'ailleurs prévue dans le modèle de dislocation à cœur étendu, qui a été développé en tenant compte de l'effet du dopage [14].

Ainsi il est montré dans ce modèle qu'il existe un effet important de ségrégation de HF dans le cœur des défauts linéaires entraînant d'une part une augmentation du rayon $r^{*}$ et d'autre part, une légère diminution de la valeur apparente de l'énergie $E$. Le premier effet serait alors à l'origine de l'accentuation de la non-linéarité après dopage ; le deuxième effet pourrait rendre compte de façon satisfaisante de l'augmentation de la vitesse $v_{\mathrm{d}}$.

Les modèles théoriques $[6,7,8]$ envisageaient aussi cette augmentation de la vitesse comme le montre la relation (1) puisque le dopage avec HF entraîne une diminution du temps de relaxation $\tau_{R}$. Mais dans le cas de nos expériences, la valeur de $\tau_{R}$ mesurée sur les mêmes échantillons par frottement intérieur diminue dans un rapport de l'ordre de 70 lors du dopage [5] alors que les mesures de vitesse des dislocations indiquent que $v_{\mathrm{d}}$ n'est multipliée que par un facteur de l'ordre de 2 . Nous disposons donc là d'une donnée 
supplémentaire s'opposant aux modèles s'appuyant sur l'hypothèse du mouvement des dislocations dans la glace, conditionné par l'orientation des liaisons hydrogènes.

En conclusion, la détermination de la vitesse des dislocations dans la glace dopée avec HF par des mesures directes en topographie $\mathrm{X}$ a permis de mettre en évidence un comportement non-linéaire des dislocations plus accentué encore que dans le cas de la glace pure et une augmentation de la vitesse des dislocations.
Nous confirmons ainsi la description des résultats obtenus par essais mécaniques. Enfin, nos données montrent de façon déterminante l'inadéquation de l'hypothèse suivant laquelle le désordre protonique serait à l'origine des barrières s'opposant aux mouvements des dislocations dans la glace; elles sont par contre cohérentes avec l'analyse reposant sur l'idée d'une extension du cœur des dislocations où l'organisation non-cristalline des molécules d'eau rendrait possibles des transitions structurales par mouvement coopératif entraînant l'avancée des défauts linéaires.

\section{Bibliographie}

[1] Jones, S. J., GILRA, N. K., Phys. and Chem. of Ice, Ed. E. Walley, S. J. Jones and L. W. Gold (Royal Society, Canada, Ottawa), 1973, p. 344.

[2] Fukuda, A., Higashi, A., Cryst. Lattice Defects 4 (1973) 203.

[3] MaI, C., C. R. Acad. Sci. Paris 282B (1976) 515.

[4] Jones, S. J., Gilra, N. K., Philos. Mag. 272 (1973) 457.

[5] Vassollle, R., Perez, J., Mai, C. and Tatibouët, J., J. Phys. Chem. Solids 38 (1977) 1297.

[6] Perez, J. Tatibouët, J., Vassoille, R. and Gobin, P. F., Philos. Mag. 5 (1975) 985.

[7] Whitworth, R. W., Paren, J. G. and Glen, J. W., Philos. Mag. 33 (1976) 409.
[8] Frost, M. J., Goodman, D. J. and Ashby, M. F., Philos. Mag. 33 (1976) 951.

[9] WHITWORTH, R. W., à paraître dans J. Glaciol. 21, 85 (1978) 000 .

[10] Perez, J., MAI, C. and VAssollle, R., à paraître dans J. Glaciol. 21, 85 (1978) 000

[11] Nakamura, T., Jones, S. J., Scientific Series $n^{\circ} 24$, Inland Waters Directorate, Environment, Canada.

[12] Jones, S. J., Glen, J. W., Philos. Mag. 19 (1969) 13.

[13] Johnston, W. G., Gilman, J. J., J. Appl. Phys. 30 (1959) 129.

[14] Perez, J., Mai, C., TatibouËt, J. and Vassoille, R. (à paraître). 